\title{
¿En qué medida los beneficios y riesgos de la externalización en el sector hotelero influyen en la adopción de esta estrategia por los directivos?
}

\author{
Tomás F. Espino-Rodríguez ${ }^{1 \mathrm{a}}$, Juan Carlos Ramírez-Fierro ${ }^{\mathrm{b}}$ \\ ${ }^{a}$ Universidad de Las Palmas de Gran Canaria, España \\ ${ }^{b}$ Universidad Oberta de Cataluña
}

doi: $10.20420 /$ eni. 2020.325

\begin{abstract}
Resumen
Este trabajo estudia el papel de la estrategia de externalización en el sector hotelero. Para ello se analiza en qué medida la propensión a externalizar del directivo está influenciada por los beneficios tácticos y estratégicos que aporta la estrategia de externalización. Asimismo, se analiza en qué medida los riesgos estratégicos determinan dicha propensión a externalizar. Los resultados indican que una percepción positiva de los beneficios tácticos por parte del directivo influye positivamente en la propensión a externalizar y que cuanto mayor es la percepción de los riesgos estratégicos menor será la propensión a externalizar del directivo.
\end{abstract}

Palabras clave: externalización, beneficios tácticos y estratégicos, riesgos.

Clasificación JEL: M11, L18.

Fuente de referencia: Espino-Rodríguez, T. F., \& Ramírez-Fierro, J. C. (2018). Managers' attitudes toward hotel outsourcing in a tourist destination. An approach from the benefits and risks perspective. Tourism Management Perspectives, 26, 143-152.

\section{Introducción}

En los años recientes la demanda turística ha evolucionado, el entorno se ha vuelto más competitivo y la competencia entre los destinos turísticos nuevos y los consolidados se ha intensificado. Las zonas turísticas compiten por adaptarse a las necesidades de los turistas, intentando proporcionar un alto nivel de calidad, lo que genera crecimiento y desarrollo del territorio. En este contexto, las empresas quieren desarrollar nuevos productos y servicios que satisfagan las necesidades de sus clientes en el menor tiempo posible a través de la innovación. Todo lo cual aumenta la dificultad del hotel para realizar todas sus actividades internamente, por lo que pueden requerir de suministradores externos para prestar el servicio hotelero (Chatzoglou \& Sarigiannidis, 2009). Leeman y Reynolds (2012) definen la externalización como el uso de empresas externas para realizar tareas que normalmente se han realizado internamente por el hotel.

La externalización es una técnica ampliamente recomendada para mejorar la posición competitiva y supone una oportunidad para incrementar la eficiencia en las empresas. La externalización representa un medio para adaptar los límites de la empresa mediante la reestructuración de sus actividades con el objetivo de estimular el crecimiento de su negocio principal (Bustinza, Arias-Aranda, \& Gutierrez, 2010). La externalización supone una opción para minimizar el problema de la complejidad hotelera, ya que muchas de las actividades del hotel pueden ser llevadas a cabo por proveedores (González, Gasco, \& Llopis, 2011). En muchas ocasiones las estrategias de externalización son impuestas a los directores de hoteles, especialmente las decisiones son tomadas por los consejos de administración de estas empresas. Sin embargo, no es aceptada en muchas ocasiones por los directivos o empleados

${ }^{1}$ Autor de correspondencia: tomasfrancisco.espino@ulpgc.es 
que gestionan esos hoteles. Estos directivos no son proclives a externalizar a menos que perciban beneficios o no perciban muchos riesgos en su uso. En este sentido, este trabajo analiza por qué viene determinada la actitud positiva o negativa hacia la externalización de los directivos de los hoteles.

\section{La externalización hotelera: beneficios y riesgos de la externalización}

Los beneficios que justifican el uso de la externalización se resumen en dos grandes grupos: el primero incluye factores relacionados con la reducción de costes y otros aspectos que tienen que ver con el corto plazo, dando lugar a los beneficios tácticos de la externalización, es decir, el enfoque económico de la externalización; y el segundo grupo de beneficios está relacionado con aspectos estratégicos de la empresa relacionados principalmente con la obtención de mejora de sus recursos y capacidades y de su ventaja competitiva (Quinn \& Hilmer, 1994).

Esta decisión de externalizar puede ser evaluada una y otra vez mediante multitud de factores (motivos) que pueden ser tanto de carácter estratégico como táctico y se relacionan con las metas y estrategias de la organización; por ejemplo, la reducción de costes, el enfoque en su negocio principal, el aseguramiento de la flexibilidad empresarial, la expansión o la obtención de una ventaja estratégica para apoyar los objetivos de negocio (Kroes \& Ghosh, 2010). En este trabajo centramos la percepción de estos beneficios tácticos en que la externalización permite al hotel la reducción de costes, ser más eficiente, cubrir las necesidades urgentes de personal e incrementar la rentabilidad del hotel.

Aunque el ahorro en costes es el motivo más común, la búsqueda de la flexibilidad, así como la obtención de ventajas estratégicas están adquiriendo una gran relevancia (Redondo-Cano \& Canet-Giner, 2010). La decisión de externalizar puede ayudar a la empresa a liberar los recursos necesarios para centrarse en los aspectos más importantes del negocio e invertir en nuevos proyectos con un mayor retorno, o en nuevas oportunidades (Powell, Tatikonda, \& Liao, 2006). Aspectos como calidad, flexibilidad o la implementación de nuevas tecnologías juegan un papel fundamental para mantener la competitividad de la empresa. Los beneficios estratégicos de la externalización están en relación con la adquisición de recursos, capacidades y habilidades que proporciona el proveedor, con mayor conocimiento y experiencia. La externalización permite el acceso a empresas especializadas que pueden añadir calidad al producto o servicio, hacerlas más flexibles, reducir su tamaño y los costes burocráticos innecesarios (Redondo-Cano \& Canet-Giner, 2010).

Por otra parte, un uso inapropiado de la externalización y un incremento en la misma, ocasionados en muchos casos por una falta de competitividad, o bien posibles deficiencias en las capacidades del suministrador, pueden originar que los hoteles inicien un proceso de declive que puede dejarlos sin las capacidades y habilidades necesarias para competir y/o ofrecer un servicio de calidad (Espino-Rodríguez, ChunLai, \& Baum, 2012).

Los riesgos pueden aparecer por ejemplo cuando se han externalizado actividades críticas (Hoecht \& Trott, 2006) que suponen un gran riesgo, como las que requieren de un contacto directo con el cliente o aquellas que pueden generar competencias básicas. Esto puede derivar en una pérdida de destrezas o habilidades para la innovación (McIvor, 2005). La externalización puede erosionar el potencial de la organización para el aprendizaje, especialmente si se externalizan aquellas actividades que son necesarias para desarrollar su negocio principal (Lei \& Hitt, 1995), o que podrían influir en la capacidad requerida para identificar y explotar nuevas oportunidades de negocio (EspinoRodríguez y otros, 2012). 
La dependencia del proveedor, por otra parte, puede dificultar la creación del conocimiento y la transferencia del mismo a través de los diferentes departamentos del hotel, al verse limitado con la externalización el surgimiento de nuevas e inesperadas soluciones que emanen de las interacciones entre las personas especializadas en diferentes actividades o departamentos (Quinn \& Hilmer, 1994).

La externalización puede suponer también una pérdida de control y autonomía en el desarrollo del servicio pues, por ejemplo, podría ocurrir que el hotel externalice actividades que el proveedor no llegue a suministrar en los términos de cantidad y calidad acordados. Llegado a este caso, y ante la intención de recuperar la actividad internamente, podemos encontrarnos ante la incapacidad de reconstruir la operación subcontratada al haber perdido las habilidades y capacidades necesarias para volver a desarrollarla internamente, sobre todo si hemos prescindido del personal experimentado.

Toda externalización supone una reestructuración y puede provocar malestar en la plantilla de trabajadores (Belcourt, 2006). Según González, Gasco, y Llopis (2005) puede ocurrir también que la situación de incertidumbre que puede generar la externalización conlleve a una bajada de productividad, desmotivación o inseguridad en el personal del departamento en dónde se va a llevar a cabo la estrategia de externalización.

\section{La percepción directiva de la externalización}

La percepción sobre la externalización que tenga un directivo es clave para explicar su propensión a externalizar. En concreto, la percepción que tenga del conjunto de ventajas e inconvenientes de la externalización condicionarán el mayor o menor uso de la estrategia. Esto es posible porque los directivos pueden percibir diferentes niveles de incertidumbre, al estar tal percepción influenciada por la base cognitiva de cada individuo y sus valores. Esas diferencias en las percepciones de los directivos son el factor clave explicativo de la existencia de distintos marcos decisionales y estrategias seguidas en un mismo entorno (Anderson \& Paine, 1975). Por tanto, las percepciones en combinación con los valores ayudan al directivo a tomar una decisión estratégica (en nuestro caso, la externalización) (Hambrick \& Mason, 1984). Partimos de la idea de que una percepción positiva del directivo en relación a las ventajas de la externalización podría tener un efecto positivo en su propensión a externalizar y, por el contrario, la percepción de los inconvenientes tendría un efecto negativo sobre dicha estrategia. De esta forma, una percepción positiva de la externalización puede influenciar a los directivos a realizar un mayor uso de la estrategia de externalización (EspinoRodríguez y otros, 2012).

\section{Metodología}

Nuestra investigación se centró en los establecimientos de sol y playa de categorías 3,4 y 5 estrellas de los municipios turísticos de San Bartolomé de Tirajana y Mogán pertenecientes a Gran Canaria. 77 directivos de hoteles participaron en el estudio. Para obtener los datos de la investigación nos dirigimos a ellos mediante una encuesta personal. A través de dicha encuesta se midió la propensión a externalizar mediante 7 ítems partiendo de la escala de Gewald y Dibben (2009), los beneficios $\mathrm{y}$ riesgos, mediante una escala multidimensional tipo Likert (de 1 a 7 posiciones) con 12 afirmaciones para los beneficios y 10 afirmaciones para los riesgos estratégicos basados en los trabajos de Bustinza y otros (2010) y Espino-Rodríguez y otros (2012).

\section{Análisis y resultados}

En la tabla 1 se muestran las 12 afirmaciones con sus valores medios de los beneficios analizados. Se puede apreciar que son cuatro los beneficios de la externalización que más se 
perciben entre los directivos, destacando del resto tanto en la media, como en la mediana y en la moda. Estos beneficios son: la externalización ayuda a cubrir necesidades más urgentes del personal, la externalización de servicios favorece la reducción de costes del hotel, la externalización permite incrementar la rentabilidad del hotel y la externalización de servicios permite ser más eficiente al hotel. Con respecto a los riesgos, en la tabla 2 se muestran las afirmaciones con sus respectivos valores medios. Los resultados indican que los principales riesgos estratégicos que perciben los directores de los hoteles son aquellos relacionados en primer lugar con la pérdida de diferenciación de los productos y servicios, seguidos de que la externalización puede dañar los resultados del hotel.

Tabla 1. Beneficios de la externalización

Beneficios de la externalización Media

Con la externalización la dirección del hotel dispone de más tiempo para realizar las tareas

En el hotel se realiza mejor nuestras tareas gracias a la externalización 2.82

\begin{tabular}{ll}
\hline $\begin{array}{l}\text { La externalización de servicios } \\
\text { permite ser más eficiente al hotel }\end{array}$ & 3.45 \\
\hline $\begin{array}{l}\text { La externalización hotelera permite al } \\
\text { hotel cubrir necesidades urgentes de }\end{array}$ & 4.53 \\
personal & \\
\hline $\begin{array}{l}\text { Que este hotel externalice servicios } \\
\text { ayuda a que el personal no } \\
\text { externalizado pueda hacer más cosas }\end{array}$ & 2.83 \\
\hline $\begin{array}{l}\text { Con la externalización de servicios, el } \\
\text { hotel puede centrarse en actividades } \\
\text { clave }\end{array}$ & 3.38 \\
\hline $\begin{array}{l}\text { Con la externalización sacamos más } \\
\text { trabajo adelante con menos esfuerzo }\end{array}$ & 2.79 \\
\hline $\begin{array}{l}\text { La externalización de servicios } \\
\text { favorece la reducción de costes del } \\
\text { hotel }\end{array}$ & 3.77 \\
\hline $\begin{array}{l}\text { La externalización de servicios } \\
\text { permite obtener trabajo cualificado y } \\
\text { con una gran experiencia }\end{array}$ & 3.10 \\
\hline $\begin{array}{l}\text { La externalización nos permite tener } \\
\text { más tiempo libre para emplearlos en } \\
\text { otras tareas }\end{array}$ & 2.84 \\
\hline $\begin{array}{l}\text { La externalización permite } \\
\text { incrementar la rentabilidad del hotel }\end{array}$ & 3.65 \\
\hline $\begin{array}{l}\text { La externalización permite } \\
\text { incrementar la calidad de los } \\
\text { servicios del hotel }\end{array}$ & 2.58 \\
\hline
\end{tabular}

Tabla 2. Riesgos de la externalización

\begin{tabular}{ll}
\hline \multicolumn{3}{c}{ Riesgos de la externalización } & Media \\
\hline $\begin{array}{l}\text { Con la subcontratación se puede } \\
\text { perder diferenciación de productos y }\end{array}$ & 4.61 \\
servicios & \\
\hline $\begin{array}{l}\text { La subcontratación puede suponer } \\
\text { una pérdida en el control de }\end{array}$ & 3.70 \\
actividades & \\
\hline $\begin{array}{l}\text { Desconocemos si la forma de trabajar } \\
\text { de las empresas de subcontratación es } \\
\text { la mejor }\end{array}$ & 3.81 \\
\hline $\begin{array}{l}\text { Desconocemos los beneficios que } \\
\text { puede suponer una subcontratación de }\end{array}$ & 2.22 \\
actividades & \\
\hline $\begin{array}{l}\text { Es difícil coordinar las actividades } \\
\text { subcontratadas con las actividades } \\
\text { realizadas por el hotel }\end{array}$ & 2.87 \\
\hline $\begin{array}{l}\text { Es difícil controlar las operaciones } \\
\text { realizadas por las empresas a las que } \\
\text { se subcontrata }\end{array}$ & 2.90 \\
\hline $\begin{array}{l}\text { Las decisiones de subcontratar suelen } \\
\text { no tener marcha atrás en el hotel }\end{array}$ & 2.95 \\
\hline $\begin{array}{l}\text { Con las decisiones de subcontratación } \\
\text { se pierde autonomía en la toma de } \\
\text { decisiones }\end{array}$ & 2.81 \\
\hline $\begin{array}{l}\text { Las decisiones de subcontratación } \\
\text { pueden perjudicar a los recursos y } \\
\text { capacidades del hotel }\end{array}$ & 3.22 \\
\hline $\begin{array}{l}\text { La subcontratación puede dañar los } \\
\text { resultados del hotel }\end{array}$ & 3.94 \\
\hline
\end{tabular}

Posteriormente realizamos un modelo de ecuaciones estructurales para establecer la relación entre los beneficios y riesgos con la propensión a externalizar. A partir de la figura 1, se observa que una percepción positiva de los beneficios tácticos por parte del directivo influye positivamente en la propensión a externalizar, $(\beta=0,339, p<0,01)$. Por otra parte, se observa que los beneficios estratégicos no influyen de forma significativa en la propensión a externalizar del directivo $(\beta=0,114, p>0,05)$. Con respecto al efecto de los inconvenientes o riesgos de la externalización percibidos por el directivo en la propensión a externalizar, los resultados confirman esta predicción $(\beta=-0,333 p<0,001)$. La variabilidad explicada conjuntamente por las tres variables que tomamos como referencia para la predicción de la propensión a externalizar del directivo explican un $39,9 \%$., lo que supone que el modelo tiene buena capacidad predictiva. 
Figura 1. Modelo estructural

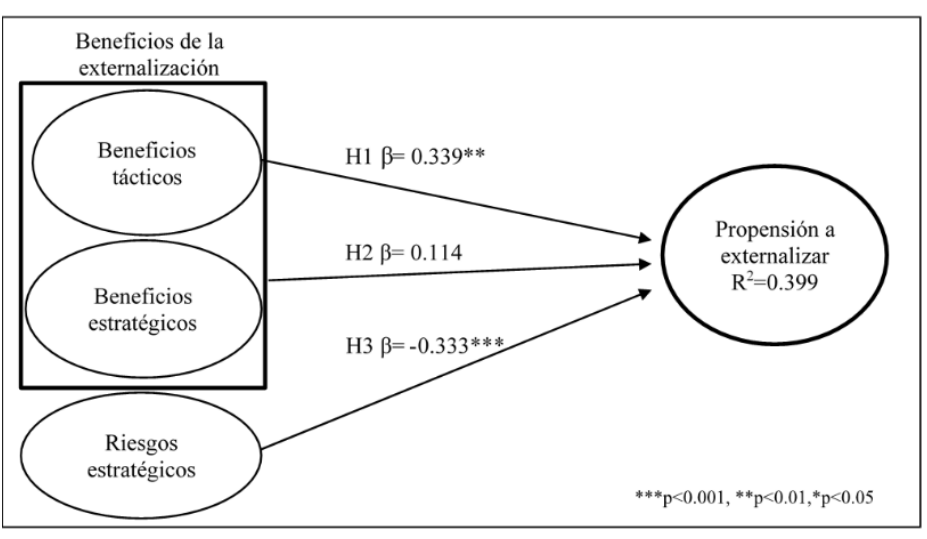

que prima la calidad, la capacidad de innovación y la capacidad de respuesta a los cambios del mercado, a un enfoque más táctico. Nuestra investigación señala un nuevo marco de la estrategia de externalización frente a otros estudios recientes en otras áreas turísticas (Espino-Rodríguez y otros, 2012) cuyos resultados establecen que la externalización permite al hotel complementar sus recursos y capacidades y acceder a un personal más cualificado y experimentado, mientras se benefician de la concentración en sus actividades clave o básicas. La externalización de los hoteles encuestados se rige por beneficios de naturaleza más táctica que estratégica. Los directivos hoteleros se rigen más por la reducción de costes que por los beneficios de la explotación de sus recursos y capacidades.

Con respecto a los inconvenientes, los resultados obtenidos avalan los obtenidos en otras investigaciones (Espino-Rodríguez y otros, 2012), siendo los principales riesgos percibidos por los directivos la pérdida de diferenciación de los productos y servicios, el desconocimiento de la forma de trabajar de los suministradores del servicio, la posible pérdida de control de las actividades y el daño que puede suponer la externalización a los resultados del hotel. En cambio, comprobamos que los directivos hoteleros no consideran irreversibles las decisiones de externalización si los resultados son insuficientes, incluso estarían dispuestos a recuperar la actividad para desarrollarla internamente de nuevo.

Por otra parte, si analizamos globalmente las ventajas y los riesgos percibidos, observamos que existe una mayor tendencia de los directivos a dar una puntuación superior a los riesgos percibidos que a las ventajas, lo que nos indica que existirá un incremento en la externalización solamente en el caso de que se consigan reducir los riesgos, ya que estos tienen un mayor peso a la hora de tomar las decisiones relacionadas con 
los límites de la empresa. El estudio demuestra que la percepción directiva de los beneficios tácticos determina la propensión a externalizar del directivo. En cambio, una percepción positiva de los beneficios estratégicos no determina de forma significativa la propensión a externalizar del directivo.

Los resultados muestran una relación negativa entre la percepción directiva de los riesgos estratégicos y la propensión a externalizar. Esta relación negativa supone que cuando los directivos perciben una mayor incertidumbre y riesgo con la externalización, más miedo tienen a dejar sus actividades en manos de terceros.

Este trabajo nos ha permitido identificar las principales ventajas o beneficios que determinan la estrategia de externalización y los principales riesgos que limitan el uso de la misma, lo que proporciona conocimiento y ayuda a los directivos hoteleros en la toma de decisiones sobre la externalización de las actividades de su hotel. Aunque nuestra investigación establece que la externalización actual está más relacionada con motivos tácticos que estratégicos, los directivos deben de ser conscientes de que la decisión de externalizar una actividad es una decisión estratégica clave, por lo que deben evitar una perspectiva a corto plazo de ahorro en costes y adoptar un enfoque a largo plazo. El planteamiento estratégico puede proporcionar a los hoteles un mejor resultado que cuando sólo se analizan los costes, pues tiene en cuenta qué tipo de actividades se deben externalizar y cuáles no para conseguir la ventaja competitiva. Esto va a permitir al hotel centrarse en aquellos procesos que realiza mejor que sus competidores o proveedores, es decir, en lo que realmente sabe hacer bien. El conocimiento de los profesionales del sector, como son los proveedores de las empresas de servicios, de los riesgos percibidos por los directivos se hace imprescindible a la hora de la realización y ejecución del contrato con la finalidad de que se pueda reducir la incertidumbre a la hora de dejar los servicios hoteleros en manos de terceros. Los directivos deben entender que si optan por externalizar actividades en sus hoteles conseguirán mayor flexibilidad y dinamismo, por lo que serán capaces de afrontar mejor los cambios y las oportunidades que presenta el sector turístico. Los profesionales del sector deben tener en cuenta que un uso inapropiado de la externalización o el incremento de la misma, puede originar un proceso de declive que puede dejar al hotel sin las capacidades y habilidades necesarias para competir.

\section{Bibliografía}

Anderson, E., \& Paine, F.T. (1975). Managerial perceptions and strategic behavior. Academy of Management Journal, 18(4), 811-823.

Belcourt, M. (2006). Outsourcing: the benefits and the risks. Human Resource Management Review, 16(2), 269-279.

Bustinza, O., Arias-Aranda, D., \& Gutierrez, L. (2010). Outsourcing, competitive capabilities and performance: an empirical study in service firms. International Journal of Production Economics, 126(2), 276-288.

Chatzoglou, P.D., \& Sarigiannidis, L. (2009). Business outsourcing and organisational performance: the case of the Greek hotel industry. International Journal of Services Technology and Management, 11, 105-127.

Espino-Rodríguez, T., Chun-Lai, P., \& Baum, T. (2012). Risks and benefits of outsourcing hotel operations: a comparison between Scotland and Taiwan. Tourism Economics, 18(1), 95-120

Gewald, H., \& Dibbern, J. (2009). Risks and benefits of business process outsourcing: A study of transaction services in the German banking industry. Information \& Management, 46, 249-257.

González, R., Gasco, J., \& Llopis, J. (2005). Information systems outsourcing risks: A study of large firms. Industrial Management \& Data Systems, 105(1), 45-62.

González, R., Gasco, J., \& Llopis, J. (2011). What do we know about outsourcing in hotels? The Service Industries Journal, 31(10), 1669-1682.

González, R., Gasco, J., \& Llopis, J. (2013). Outsourcing and strategy in Spanish town halls: a field study. Management Decision, 51(1), 97-119. 
Hambrick, D.C., \& Mason, P.A. (1984). Upper Echelons: The Organization as a Reflection of its Top Managers. Academy or Management Review, 9(2), 193-206.

Hoecht, A., \& Trott, P. (2006). Innovation risks of strategic outsourcing. Technovation, 26, 672-681.

Jiang, B., Frazier, G., \& Prater, E. (2006). Outsourcing effects on firms' operational performance: an empirical study. International Journal of Operations and Production Management, 26, 1280-1300.

Kroes, J., \& Ghosh, S. (2010). Outsourcing congruence with competitive priorities: impact on supply chain and firm performance. Journal of Operations Management, 28, 124-143.

Leeman, D., \& Reynolds, D. (2012). Trust and outsourcing. Do perceptions of trust influence the retention of outsourcing providers in the hospitality industry? International Journal of Hospitality Management, 31, 601-608.

Lei, D., \& Hitt, M. (1995).Strategic restructuring and outsourcing: The effect of mergers and acquisitions and LBOs on building firm skills and capabilities. Journal of Management, 21(5), 835-859.

McIvor, R. (2005). The Outsourcing Process. Strategies for Evaluation and Management. Cambridge University Press, Cambridge.

Powell, S., Tatikonda, M., \& Liao, Y. (2006). A behavioural study of supply manager decisionmaking: factors influencing make versus buy evaluation. Journal of Operations Management, 24 (6), 822-838.

Quinn, J., \& Hilmer, F. (1994). Strategic outsourcing. Sloan Management Review, 35 (4), 43-55.

Redondo-Cano, A., \& Canet-Giner, M.T., (2010). Oursourcing agrochemical services: economic or strategic logic? Service Business, 4, 237-252. 